\title{
QUALIDADE DO PREENCHIMENTO DE ATESTADOS DE ÓBITOS DE MENORES DE UM ANO NA REGIÃO METROPOLITANA DO RIO DE JANEIRO*
}

\author{
Flávia Maria Leal Niobey** \\ Angela Maria Cascão*** \\ Milena Piraccini Duchiade ${ }^{* * *}$ \\ Paulo Chagastelles Sabroza*
}

NIOBEY, F. M. L. et al. Qualidade do preenchimento de atestados de óbitos de menores de um ano na região metropolitana do Rio de Janeiro. Rev. Saúde públ., S. Paulo, 24: 311-8, 1990.

RESUMO: No âmbito de uma investigação sobre os determinantes de mortalidade infantil na região metropolitana do Rio de Janeiro, RJ (Brasil) durante um ano, foram estudadas e comparadas as declarações de óbito com as informações disponíveis nos prontuários e boletins hospitalares, de uma amostra de crianças. Objetivou-se avaliar a qualidade do preenchimento dessas declarações para alertar os profissionais de saúde para sua utilização como documento gerador de dados para elaboração e avaliação de programas de saúde e não apenas como exigência legal para o sepultamento. Apenas $52,3 \%$ das declarações mantiveram a mesma causa básica após consulta ao prontuário. A necrópsia foi realizada em apenas $42,8 \%$ dos óbitos neonatais e $71,5 \%$ dos pós-neonatais, ocorridos fora do ambiente hospitalar. Foram avaliados: idade e grau de instrução da māe, intervalo de tempo e exame complementar, peso ao nascer e necrópsia, cujo grau de preenchimento das declaraçōes ficou muito aquém do necessário, evidenciando as limitações das estatísticas oficiais de mortalidade que se baseiam nessas informações.

DESCRITORES: Atestados de óbitos. Registros médicos. Causa de morte.

\section{INTRODUÇÃO}

De maneira geral, a declaração de óbito é vista pelo médico apenas como uma exigência legal para o sepultamento, sendo raras vezes encarada como fonte geradora de dados sobre a saúde de uma populaçăo. Embora a causa básica de morte tenha sido definida com precisão na Assembléia Mundial da Saúde de 1948, e desde 1976 tenha sido instituído um modelo único de declaração de óbito pelo Ministério da Saúde, o seu preenchimento continua apresentando inúmeros problemas. Vários trabalhos em que se avalia a qualidade desse preenchimento vêm sendo publicados ${ }^{2,3,5,7,17} \mathrm{e}$ têm apontado deficiências.

Apesar do valor inegável das estatísticas oficiais de mortalidade, devemos atentar para as suas limitaçōes, uma vez que são construídas com os dados retirados das declarações de b́bito.

Além da causa básica de morte - de importância fundamental - todos os outros itens constantes das declaraçס̃es de óbito também são mal informados, afetando o agrupamento por idade $e$ quaisquer outros estudos que se pretenda realizar.
Assim, por exemplo, enquanto na Grã-Bretanha e nos EUA, estudos clássicos baseados em informaçōes diretas das declarações de óbito há muito tempo já revelam relações entre ocupação, estrato social e taxas diferenciadas de mortalidade ${ }^{1,13}$, no Brasil esses ainda aparecem em pequeno número.

A repercussão da carência de informações das declarações de óbito nas estatísticas oficiais não costuma ser medida, gerando conformismo e acomodação entre os médicos, que não se sentem responsáveis pelo preenchimento da parte "menos nobre" da declaração de óbito, referente ao nome do falecido, sua profissão, idade, residência, preocupando-se apenas com o atestado médico (parte IV), ficando para funcionários administrativos ainda menos comprometidos - a tarefa de completar as demais partes.

Essa falta de esclarecimentos de todos os profissionais que manipulam as declaraçōes de óbito leva a um preenchimento deficiente, inviabilizando posterior aproveitamento pleno das informaçōes, o que obriga os profissionais de saúde pública a contornar este problema através da realização de inquéritos especiais, muito mais demo-

* Pesquisa financiada pela FINEP (Financiadora de Estudos e Projetos) e INAMPS (Instituto Nacional de Assistência Médica e Previdência Social) - Convênio $n^{2} 43.860 .197 .00$

* Escola Nacional de Saúde Pública/FIOCRUZ - Av. Leopoldo Bulhōes, 1480 - Manguinhos - 21041 - Rio de Janeiro, RJ - Brasil.

* * Secretaria Estadual de Saúde - Rua México, 128 - sala 820 - Castelo — 20030 - Rio de Janeiro, RJ - Brasil. 
rados e dispendiosos e, portanto, de abrangência menor $^{14,18,19}$.

Estes problemas assumem especial relevância em nosso país, onde as estatísticas de mortalidade constituem-se na principal fonte de dados para os diagnósticos de saúde.

O modelo único de declaração de óbito introduzido pelo Ministério da Saúde, em 1976, consta de 8 partes (partes I a VIII) ${ }^{8,10}$. A parte IV é o Atestado Médico, composto por 5 itens: o item 32 referese à assistência médica durante a doença que ocasionou a morte; o 33 refere-se à qualificação do médico atestante e o 34 à confirmação do diagnóstico. Os itens 35 e 36 assumem especial importância pois é onde a causa de morte é declarada. Essa declaração é feita no "Modelo Internacional de Certificado Médico da Causa da Morte", recomendado pela Assembléia Mundial de Saúde de $1948^{\circ}$.

No item 35 (parte 1), deverá ser registrada a causa básica do óbito, definida como: a) doença ou lesão que iniciou a cadeia de acontecimentos patológicos que conduziram diretamente à morte; ou b) circunstâncias do acidente ou violência que produziram a lesão fatal. No item 36 (parte II), deverão ser registradas as causas contribuintes, se presentes.

Ainda nos itens 35 e 36 há o espaço reservado ao intervalo de tempo que duraram as doenças citadas.

De grande valia para o conhecimento da mortalidade infantil é o preenchimento da parte III da declaração de b́bito: dados sócio- econốmicos e biológicos dos pais (itens $21,22,23,24,25$ ), número de filhos tidos (item 26), dados da gestação (itens 27 e 28), dados do parto (item 29), morte do feto (item 30 ) e peso ao nascer (item 31).

Desde a Investigação Interamericana sobre a mortalidade na infấncia 14 , sabe-se que o peso ao nascer é um dos principais fatores de risco que influem na mortalidade infantil, dado este confirmado pelos inúmeros estudos posteriores $6,15,18,20$.

Do mesmo modo, diversos estudos têm comprovado a importância do grau de instrução materna enquanto determinante da mortalidade infantil 4, 12, 16. Estas informaçőes, entretanto, freqüentemente deixam de ser adequadamente preenchidas.

Desde a introdução, em 1976, do modelo pa- dronizado da declaração de óbito pelo Ministério da Saúde, no Brasil, o Centro Brasileiro para a Classificação de Doenças* vem desenvolvendo paciente trabalho de educação no meio médico, divulgando normas ${ }^{8}$ e reciclando os profissionais interessados.

Após dez anos desse trabalho, trata-se de verificar até que ponto este esforço tem sido bem sucedido no Estado do Rio de Janeiro, segundo maior centro urbano do Brasil, sede de 13 escolas médicas, segundo informação fornecida pela Associação Brasileira de Escolas Médicas.

No âmbito de uma investigação sobre os determinantes da mortalidade infantil na região metropolitana do Rio de Janeiro, desenvolvida conjuntamente pela Escola Nacional de Saúde Pública da FIOCRUZ e pela Secretaria Estadual de Saúde do Rio de Janeiro, e ainda em curso, foi possível comparar as declaraçōes de óbito originais de uma amostra de crianças com as informações disponíveis nos prontuários e boletins hospitalares das mesmas. Tal trabalho constitui-se num dos primeiros resultados dessa investigação.

Assim, pois, pretende-se fazer uma avaliação das falhas do preenchimento de declaraçðes de óbitos ocorridos na regiāo metropolitana do Rio de Janeiro, nos itens reservados à causa básica do óbito, causas contribuintes, necrópsia, intervalo de tempo, idade da mãe e grau de instrução da mãe e também fazer uma análise, em separado, dos obitos nos quais foram realizadas a necrópsia.

\section{METODOLOGIA}

As declarações de 6 bito que serão avaliadas no presente trabalho correspondem a uma amostra de $25 \%$ (6.672) do total de óbitos de menores de um ano ocorridos na região metropolitana do Rio de Janeiro, no período de maio de 1986 a abril de 1987.

Os óbitos de menores de 28 dias (neonatais) representam mais da metade dos óbitos de menores de um ano, mas tinha-se interesse em analisar os determinantes da mortalidade infantil sobretudo para os óbitos de crianças entre 29 dias e 11 meses (pós-neonatais). Por isso, optou-se por um processo amostral que resultasse em maior probabilidade a esses óbitos de participarem do estudo em relação aos neonatais. Dessa forma, foi feita uma amostra estratificada representando $15 \%$ dos óbitos neonatais de $40 \%$ dos pós-neonatais. Ao longo dos 12 meses do estudo, a soma desses dois grupos foi de 1.703 óbitos ( 1.178 pós-neonatais e 525 neonatais). 
Foram excluídos do estudo 517 b́bitos ocorridos nas áreas de Magé, Itaboraí, Maricá, Itaguaí, Paracambi e Mangaratiba por serem áreas de difícil acesso, além de representarem um percentual pequeno em relação ao total $(7,6 \%)$.

Para fins do projeto, a amostra foi dividida em cinco grandes grupos, baseados na causa básica do 6́bito codificada segundo critérios da Classificação Internacional de Doenças" a saber: Causas Perinatais (CID: 760 a 779), Diarréias (CID: 001 a 009), Pneumonias (CID: 480 a 486), Desnutrição (CID: 260 a 269) e Outras Causas (as demais).

A partir dessa amostra a equipe de médicos do projeto realizou consulta aos prontuários hospitalares de cada uma das crianças, o que levou ao preenchimento de um questionário e de uma "nova" declaração de óbito, segundo critérios divulgados pelo Centro Brasileiro de Classificação de Doenças. A nova causa básica foi então recodificada, confirmando ou não a causa básica original, preenchida pelo médico que atestou o óbito.

Além da entrevista hospitalar, foram também realizadas entrevistas no domicílio, levando ao preenchimento de um outro questionário baseado apenas nas informações obtidas com a mãe ou familiares da criança.

Vale ressaltar que as "novas" declarações de óbito foram preenchidas com base apenas nas informaçōes obtidas através do prontuário hospitalar sem levar em conta os dados do questionário domiciliar.

Dentre os óbitos sorteados, 3 tiveram que ser excluídos da análise após iniciado o projeto por que continham informaç̋es erradas na declaração de óbito - a saber: o primeiro era uma criança que faleceu com mais de um ano de vida, a segunda era moradora do Município de Cabo Frio (RJ) e a terceira era moradora do Mato Grosso do Sul. É importante esclarecer que essas informaçōes foram descobertas somente após a ida da equipe ao hospital onde constavam o endereço e a idade corretos.

Dos 1.700 óbitos restantes, 160 (146 pós e 14 neonatais) ocorreram fora do hospital (item 10 da Declaração de Óbito - local de ocorrência do óbito) em domicílio, em via pública e em delegacia. Nenhum óbito teve seu local de ocorrência ignorado. Esses foram denominados óbitos extrahospitalares.

Dessa forma, para consulta ao prontuário hospitalar, restaram 1.543 óbitos registrados na declaração de óbito como ocorridos no hospital. Dentre esses, dois grupos permaneceram sem essa consulta. O primeiro foi de "perdas hospitalares" - óbitos em que o prontuário não foi localizado no hospital. O segundo grupo foi por nós denominado "falso-hospitalares" - aqueles em que após a ida ao hospital, a equipe descobriu que as crianças chegaram já sem vida ao serviço de saúde. Os óbitos só eram considerados deste grupo quando havia algum registro no hospital de que a criança já tinha dado entrada cadáver ou quando não havia nenhum registro de entrada no serviço, porém a declaração de óbito era fornecida pelo Instituto Médico-Legal e a história da mãe confirmava a suspeita. Tal grupo foi objeto de um estudo em separado, atualmente em fase de publicação.

Assim sendo, os óbitos da amostra ficaram distribuídos como mostra a Tabela 1.

\section{TABELA 1}

Distribuição da amostra de óbitos de menores de um ano ocorridos na região metropolitana do Rio de Janeiro, no Período de Maio/86 a Abril/87.

\begin{tabular}{lccccc}
\hline & \multicolumn{5}{c}{ Neonatais Pós-Neonatais } \\
& $\mathrm{N} \propto$ & $\%$ & $\mathrm{~N} \varrho$ & $\%$ \\
\hline $\begin{array}{l}\text { Obitos } \\
\text { Obitos que tiveram }\end{array}$ & 461 & 87,8 & 806 & 68,4 \\
Obitos falso-hospitalares & 07 & 1,3 & 124 & 10,5 \\
Obitos extra-hospitalares & 14 & 2,7 & 146 & 12,4 \\
Obitos fora da amostra & - & - & 03 & 0,3 \\
Perdas hospitalares & 43 & 8,2 & 99 & 8,4 \\
\hline \multicolumn{1}{c}{ Total } & 525 & 100,0 & 1.178 & 100,0 \\
\hline
\end{tabular}

Seguindo orientação de Fonseca e Laurenti ${ }^{3}$, foram considerados completos os atestados em que estavam preenchidos os itens intervalo de tempo e exame complementar, além da parte I. Foram considerados completos (com seqüência), aqueles que apresentavam uma sequência causal lógica na parte I e, da mesma forma, completos sem seqüência, aqueles que não a apresentavam. Dentre os considerados completos, foi verificada ainda a presença ou não de informaçăo na parte II.

Os atestados considerados incompletos foram aqueles que não apresentavam preenchido o item intervalo de tempo, exame complementar ou ambos; esses foram também divididos em: com seqüência e sem seqüência.

Foram verificados ainda, separadamente, a proporção de declaraçōes de óbito que tinham preenchidas o intervalo de tempo, o exame complementar, a necrópsia, a idade da mãe e o grau de instrução e também aqueles que não possuíam as informações de intervalo de tempo e exame complementar simultaneamente. 
Foram considerados atestados corretos aqueles que apresentaram a mesma causa básica nos atestados originais após consulta aos prontuários médicos. Se a causa básica foi registrada no atestado em seqüência lógica, foi considerado correto no original; se a causa básica foi citada fora de seqüência, mas se foi selecionada após a aplicação das "Regras de Seleção da Causa Básica de Mortalidade", foi considerado correto após as regras.

Se o atestado não informava a verdadeira causa básica do óbito, foi considerado incorreto e, se a causa básica era citada porém não era selecionada após a aplicaçăo das regras, $o$ atestado foi considerado incorreto mas com a verdadeira causa básica citada.

Cabe lembrar que 433 óbitos $(25,5 \%)$ não puderam ser analisados se corretos ou incorretos por tratar-se daqueles grupos onde o prontuário médico não foi consultado (perdas hospitalares, 6bitos extra-hospitalares e falso-hospitalares).

Quanto ao item necrópsia, foi apurado o número de óbitos que foram à necrópsia e por quais instituições.

\section{RESULTADOS E DISCUSSÃO}

\section{Preenchimento da Parte IV - Atestado Médico}

Das 806 declaraç̃̃es de 6́bitos pós-neonatais, verificou-se que $52,3 \%$ apresentaram-se corretas $(45,4 \%$ no original e $6,9 \%$ após aplicação das regras de Classificação da Causa Básica do Óbito) ${ }^{9}$. Nas declarações de óbitos neonatais, foram encontrados $53,1 \%$ incorretas (Tabela 2).

\section{TABELA 2}

Distribuição da amostra de declaraçðes de óbitos infantis, segundo classificação de Corretos e Incorretos, ocorridos na regiäo metropolitana do Rio de Janeiro, no período de maio/86 a abril $/ 87$

\begin{tabular}{lccccc}
\hline \multirow{2}{*}{ Declaração de Óbito } & \multicolumn{2}{c}{ Neonatais } & \multicolumn{2}{c}{ Pós-Neonatais } \\
\cline { 2 - 5 } & $\mathrm{N}^{2}$ & $\%$ & $\mathrm{~N}^{\circ}$ & $\%$ \\
\hline Corretos no original & 94 & 20,3 & 366 & 45,4 \\
Corretos após as regras & 74 & 16,0 & 56 & 6,9 \\
Incorretos & 244 & 53,1 & 257 & 31,9 \\
$\begin{array}{l}\text { Incorretos, mas com a causa } \\
\text { básica declarada }\end{array}$ & 49 & 10,6 & 127 & 15,7 \\
\hline \multicolumn{1}{c}{ Total } & 461 & 100,0 & 806 & 100,0 \\
\hline
\end{tabular}

Nota: Foram excluídos 131 obitos falso-hospitalares, 160 6bitos extra-hospitalares, 142 perdas de prontuário hospitalar e 3 óbitos fora da amostra.

O percentual de declarações incorretas, porém com a verdadeira causa básica citada, embora não na ordem adequada, foi de $15,7 \%$ para os pósneonatais e $10,6 \%$ para os neonatais. Isso mostra que, caso se apurassem as causas múltiplas de morte, existentes nos atestados, como é proposto através do sistema ACME*, por exemplo, a informação sobre a contribuição destas causas poderia ser recuperada.

Observamos que, dos 525 6́bitos neonatais, apenas 24 declaraçōes $(4,6 \%)$ apresentavam-se completas e nos pós-neonatais, a mesma situação se repete com $52(4,4 \%)$ completas (ver Tabela 3 ).

\section{TABELA 3}

Distribuição da amostra de declaraçōes de óbitos ocorridos na região metropolitana do Rio de Janeiro, no período de maio/86 a abril/87, segundo classificação de completos e incompletos.

\begin{tabular}{|c|c|c|c|c|c|c|c|c|c|c|c|}
\hline \multicolumn{2}{|c|}{ Declaração de Óbito } & \multicolumn{2}{|c|}{$\begin{array}{l}\text { C/Seqüuência Ló } \\
\text { gica na Parte I }\end{array}$} & \multicolumn{2}{|c|}{$\begin{array}{l}\text { S/Seqüência Ló- } \\
\text { gica na Parte I }\end{array}$} & \multicolumn{2}{|c|}{$\begin{array}{c}\text { C/Informaçāo } \\
\text { na Parte I I }\end{array}$} & \multicolumn{2}{|c|}{$\begin{array}{c}\text { S/Informação } \\
\text { na Parte I I }\end{array}$} & \multicolumn{2}{|c|}{ Total } \\
\hline & & $N^{2}$ & $\%$ & $N^{2}$ & $\%$ & $\mathrm{~N}^{\circ}$ & $\%$ & $\mathrm{~N}^{2}$ & $\%$ & $\mathrm{~N}^{\circ}$ & $\%$ \\
\hline \multirow{2}{*}{ Completa } & Neonatais & 22 & 91,7 & 2 & 8,3 & 3 & 12,5 & 21 & 87,5 & 24 & 100,0 \\
\hline & Pós-neonatais & 39 & 75,0 & 13 & 25,0 & 11 & 21,1 & 41 & 78,9 & 52 & 100,0 \\
\hline \multirow{2}{*}{ Incompleta } & Neonatais & 363 & 72,5 & 138 & 27,5 & & & & & 501 & 100,0 \\
\hline & Pós-neonatais & 873 & 77,7 & 250 & 22,3 & & & & & 1.123 & 100,0 \\
\hline
\end{tabular}

\footnotetext{
* $\quad$ "Automated Classification of Medical Entities".
} 
A Tabela 4 mostra que grande parte das declarações de óbito não apresenta os itens intervalo de tempo e exame complementar preenchidos $(39,4 \%$ para os neonatais e $45,8 \%$ para os pós-neonatais) e só uma pequena parte apresenta ambos preenchidos $(4,6 \%$ para os neonatais e $4,5 \%$ para os pósneonatais).

Cabe ressaltar que, embora esses dados a rigor não interfiram nas estatísticas oficiais de mortalidade, a negligência no seu preenchimento mostra, mais uma vez, que é um documento não reconhecido como fonte geradora de dados.

Optou-se por analisar separadamente os 6bitos pelo seu local de ocorrência "de fato" para melhor avaliação, nas diversas situaçōes, do procedimento da necrópsia. Convém esclarecer que nem todos os óbitos, que foram à necrópsia, apresentavam o item preenchido; muitas vezes essa identificação foi feita pelo carimbo do Instituto Médico Legal (IML) ou do médico-legista que assinou o laudo ou ainda pela informação obtida no prontuário hospitalar (no caso dos óbitos hospitalares).
A Tabela 5 corresponde ao preenchimento do item necrópsia. Verifica-se que tanto nos óbitos hospitalares quanto nos extra-hospitalares o percentual de não-preenchimento é muito alto $(41,3 \%$ nos neonatais e $48,2 \%$ nos pos-neonatais hospitalares e $71,4 \%$ nos neonatais e $41,1 \%$ nos posneonatais extra-hospitalares). Era de se esperar que os óbitos hospitalares, cujas declaraçōes de óbito foram preenchidas no hospital em que a criança esteve internada, apresentassem um percentual mais baixo de omissão de informações, mas essa análise em separado revelou que isso não ocorre, pelo menos no que se refere ao item necrópsia.

Pela Tabela 6 verifica-se que dos 1.146 óbitos hospitalares pós-neonatais, que continham essa informação, apenas $11,8 \%$ foi à necrópsia; dos 124 falso-hospitalares, $81,5 \%$ e dos 146 extrahospitalares, $63,0 \%$. Dentre os neonatais, observou-se uma situação semelhante com $13,3 \%$ dos 6́bitos hospitalares indo à necrópsia e $85,7 \%$ e $21,4 \%$ dos "falso-hospitalares" e extrahospitalares, respectivamente.

\section{TABELA 4}

Distribuição da amostra de declaraçāes de óbitos de menores de um ano, segundo preenchimento dos itens intervalo de tempo e exame complementar, na região metropolitana do Rio de Janeiro, no período de maio/86 a abril/87

\begin{tabular}{lccccccccc}
\hline & \multicolumn{9}{c}{ Exame Complementar } \\
Intervalo de Tempo & \multicolumn{3}{c}{ Neonatais } & \multicolumn{2}{c}{ Sub-total } & \multicolumn{2}{c}{ Sim } & \multicolumn{2}{c}{ Sub-total } \\
& Nim & $\%$ & Ne & $\%$ & $\mathrm{~N}^{2}$ & $\%$ & $\mathrm{~N}^{\text {e }}$ & $\%$ \\
\hline Sim & 24 & 4,6 & 31 & 5,9 & 53 & 4,5 & 68 & 5,8 \\
Não & 287 & 54,7 & 494 & 94,1 & 569 & 48,4 & 1.107 & 94,2 \\
\hline Total & 311 & 59,2 & 525 & 100,0 & 622 & 52,9 & 1.175 & 100,0 \\
\hline
\end{tabular}

\section{TABELA 5}

Distribuição da amostra de declarações de óbitos de menores de um ano, segundo preenchimento do item necrópsia, ocorridos na região metropolitana do Rio de Janeiro, no período de maio/86 a abril/87.

\begin{tabular}{|c|c|c|c|c|c|c|c|c|}
\hline \multirow{4}{*}{$\begin{array}{l}\text { Local de } \\
\text { Ocorrência }\end{array}$} & \multicolumn{8}{|c|}{ Preenchimento do Item Necrópsia } \\
\hline & \multicolumn{4}{|c|}{ Neonatais } & \multicolumn{4}{|c|}{ Pós-Neonatais } \\
\hline & \multicolumn{2}{|c|}{ Sim } & \multicolumn{2}{|c|}{ Sub-total } & \multicolumn{2}{|c|}{$\operatorname{Sim}$} & \multicolumn{2}{|c|}{ Sub-total } \\
\hline & $\mathrm{N}^{\circ}$ & $\%$ & $N^{2}$ & $\%$ & $\mathrm{~N}^{2}$ & $\%$ & $N^{2}$ & $\%$ \\
\hline Óbitos-Hospitalares & 296 & 58,7 & 504 & 100,0 & 469 & 51,8 & 905 & 100,0 \\
\hline Óbitos Falso-Hospitalares & 5 & 71,4 & 7 & 100,0 & 90 & 72,6 & 124 & 100,0 \\
\hline Óbitos Extra-Hospitalares & 4 & 28,6 & 14 & 100,0 & 86 & 58,9 & 146 & 100,0 \\
\hline Total & 305 & 58,1 & 525 & 100,0 & 645 & 54,9 & 1.175 & 100,0 \\
\hline
\end{tabular}




\section{TABELA 6}

Distrisbuição da amostra de declaraçōes de óbitos de menores de um ano, segundo realização da necrópsia, ocorridos na região metropolitana do Rio de Janeiro, no período de maio/86 a abril/87.

\begin{tabular}{|c|c|c|c|c|c|c|c|c|}
\hline \multirow{4}{*}{$\begin{array}{l}\text { Local de } \\
\text { Ocorrência }\end{array}$} & \multicolumn{8}{|c|}{ Realização da Necrópsia } \\
\hline & \multicolumn{4}{|c|}{ Neonatais } & \multicolumn{4}{|c|}{ Pós-Neonatais } \\
\hline & \multicolumn{2}{|c|}{ Sim } & \multicolumn{2}{|c|}{ Sub-total } & \multicolumn{2}{|c|}{ Sim } & \multicolumn{2}{|c|}{ Sub-total } \\
\hline & $\mathrm{N}^{\mathrm{e}}$ & $\%$ & $N^{2}$ & $\%$ & $\mathrm{~N}^{2}$ & $\%$ & $\mathrm{~N}^{\mathrm{e}}$ & $\%$ \\
\hline Óbitos-Hospitalares & 35 & 13,3 & 263 & 100,0 & 135 & 11,8 & 1.146 & 100,0 \\
\hline Óbitos Falso-Hospitalares & 6 & 85,7 & 7 & 100,0 & 101 & 81,5 & 124 & 100,0 \\
\hline Óbitos Extra-Hospitalares & 3 & 21,4 & 14 & 100,0 & 92 & 63,0 & 146 & 100,0 \\
\hline Total & 44 & 15,5 & 284 & 100,0 & 328 & 23,2 & 1.416 & 100,0 \\
\hline
\end{tabular}

TABELA 7

Distribuição da amostra de declarações de óbitos de menores de um ano ocorridos na região metropolitana do Rio de Janeiro, no período de maio/86 a abril/87, segundo local da realizaçăo da necrópsia

\begin{tabular}{|c|c|c|c|c|c|c|c|c|c|c|c|c|}
\hline \multirow{4}{*}{$\begin{array}{l}\text { Local do } \\
\text { Óbito }\end{array}$} & \multicolumn{12}{|c|}{ Local da Necrópsia } \\
\hline & \multicolumn{6}{|c|}{ Neonatais } & \multicolumn{6}{|c|}{ Pós-Neonatais } \\
\hline & \multicolumn{2}{|c|}{ IML } & \multicolumn{2}{|c|}{ Hospitais } & \multicolumn{2}{|c|}{ Sub-Total } & \multicolumn{2}{|c|}{ IML } & \multicolumn{2}{|c|}{ Hospitais } & \multicolumn{2}{|c|}{ Sub-Total } \\
\hline & $\mathrm{N}^{2}$ & $\%$ & $\mathrm{~N}^{\mathrm{e}}$ & $\%$ & $N^{2}$ & $\%$ & $N^{0}$ & $\%$ & $N^{0}$ & $\%$ & $\mathrm{~N}^{0}$ & $\%$ \\
\hline Hospitalar & 1 & 2,9 & 34 & 97,1 & 35 & 100,0 & 42 & 31,1 & 93 & 68,9 & 135 & 100,0 \\
\hline Falso-Hospitalar & 6 & 100,0 & - & - & 6 & 100,0 & 99 & 98,0 & 2 & 2,0 & 101 & 100,0 \\
\hline Extra-Hospitalar & 3 & 100,0 & - & - & 3 & 100,0 & 92 & 100,0 & - & - & 92 & 100,0 \\
\hline Total & 10 & 22,7 & 34 & 77,3 & 44 & 100,0 & 233 & 71,0 & 95 & 29,0 & 328 & 100,0 \\
\hline
\end{tabular}

I M L= Instituto Médico Legal

\section{TABELA 8}

Distribuição da amostra de declaraçőes de óbitos de menores de um ano, segundo preenchimento dos itens idade da mãe e grau de instrução da mãe, na região metropolitana do Rio de Janeiro, no período de maio/86 a abril/87.

\begin{tabular}{|c|c|c|c|c|c|c|c|c|}
\hline \multirow{3}{*}{$\begin{array}{c}\text { Declaraçăo } \\
\text { de } \\
\text { Óbito }\end{array}$} & \multicolumn{4}{|c|}{ Neonatais } & \multicolumn{4}{|c|}{ Pós-Neonatais } \\
\hline & \multicolumn{2}{|c|}{ Sim } & \multicolumn{2}{|c|}{ Sub-total } & \multicolumn{2}{|c|}{ Sim } & \multicolumn{2}{|c|}{ Sub-total } \\
\hline & $\mathrm{N}^{2}$ & $\%$ & $N^{2}$ & $\%$ & $\mathrm{~N}^{\circ}$ & $\%$ & $N^{2}$ & $\%$ \\
\hline Idade da Mãe & 359 & 68,4 & 525 & 100,0 & 623 & 53,0 & 1.175 & 100,0 \\
\hline Grau de Instrução da Mãe & 319 & 60,8 & 525 & 100,0 & 517 & 44,0 & 1.175 & 100,0 \\
\hline
\end{tabular}

A Tabela 7 mostra que, o IML aparece, em grande parte, como responsável pelas necrópsias. Isso ocorre porque o Estado do Rio de Janeiro não dispõe de um Serviço de Verficação de Óbitos organizado, o que leva à uma sobrecarga do Instituto que só deveria realizar a necrópsia no caso de óbitos por causas externas. Os resultados dessas necrópsias decorrem, em geral, de exames macroscópicos que evidenciam a causa terminal, não sendo suficientes para determinação de causas básicas nos casos de mortes não-violentas.

A necrópsia dessas crianças internadas, algumas vezes são desenvolvidas pelos serviços de 
patologia de alguns hospitais que têm interesse em realizá-las. Assim sendo, os médicos dos demais hospitais vêem-se apenas com a opção de enviar os óbitos sem diagnóstico definido ao IML.

Vale ainda comentar os ditos óbitos "falso-hospitalares". Essas declarações de obito são uma situação particular, porque além de apresentarem as falhas de preenchimento comuns às outras, são também errôneas em um de seus aspectos mais óbvios que é o registro correto do local de ocorrência. Embora a criança já esteja falecida ao dar entrada no hospital, a declaração de óbito registra essa morte como ocorrida no mesmo.

Preenchimento da Parte III - Destinada aos Óbitos de Menores de um ano e Óbitos Fetais.

Observa-se na Tabela 8 que os itens idade e grau de instrução da mãe são mais preenchidos nas declaraçōes de óbitos neonatais do que nas pósneonatais. Isso mostra que existe maior preocupação de registrar dados referentes à mãe quando se trata de óbitos de menores de 28 dias, devida à presença de dados no prontuário da gestante.

A avaliação do item peso ao nascer (que somente deve ser preenchido para menores de 28 dias) mostrou um percentual mais alto de preenchimento $(67,2 \%)$.

\section{CONCLUSÕES}

A partir dos presentes resultados pode-se concluir que apesar do Sistema Nacional de Mortalidade, implantado já há mais de dez anos, permanece existindo falta de informação no preenchimento das declarações de óbito, refletindo o desconhecimento dos profissionais quanto à importância desta enquanto documento de estatística vital, fundamental para construção de estatísticas de saúde fidedignas, capazes de fornecerem subsídio confiável para elaboração e avaliação de programas de saúde.

Os problemas nesse preenchimento aparecem com clareza em todos os itens existentes na declaração de óbito, desde os dados de identificação, história gestacional da mãe, até os dados da causa da morte.

E importante reforçar que a falta de um serviço de Verificação de Óbitos oficial leva os hospitais a ficarem sem opção, tendo que enviar seus óbitos sem diagnósticos ao IML, onde permanecem não esclarecidos.

Fato da maior importância é a falha no preenchimento da parte IV (Atestado Médico); muitas vezes a doença que deu origem aos eventos que levaram à morte não é nem mesmo mencionada.

Permanece a necessidade de trabalhar com os médicos, de modo a ressaltar a importância de seu papel enquanto elo inicial da cadeia de informaçōes que viabilizam as estatísticas vitais. A discussão dos atestados de óbitos referentes aos casos em estudo nas sessőes clínicas dos hospitais, sejam eles universitários ou não, como já se faz em alguns locais, é um instrumento útil de reciclagem e conscientização dos profissionais de saúde. Para tanto, seria preciso que os hospitais mantivessem uma cópia dos atestados por eles emitidos, o que muitas vezes não é feito.

A meta de aproveitar plenamente todas as informaçōes constantes do atestado de óbito é factível a médio prazo bastando, para tanto, que se desenvolva um esforço no sentido de mobilizar os médicos, convencendo-os da importância de seu papel.

NIOBEY, F. M. L. et al. [The quality of the filling - in of death certificates of children below one year of age in the metropolitan region of Rio de Janeiro. Rev. Saúde públ., S. Paulo, 24: 311-8, 1990.

ABSTRACT: Within the scope of an investigation into infant mortality determinants in the metropolitan region of Rio de Janeiro during one-year period, the original death certificates of a sample of children were studied and compared with information provided on them by hospital case-histories and records. This was done with a view to assessing the quality of the filling in of certificates for the purpose of calling the attention of health officers to their use as documents furnishing data for the preparation and evaluation of health programmes and note simply as a legal requirement for burying. Only $52.3 \%$ of the basic causes given on the certificates were maintened after the examination of the case histories. Necropsy was carried out on only $42.8 \%$ of those neonatal deaths and $21.5 \%$ of post-neonatal deaths which took place outside the hospital. Other items that were evaluated included mother's age and education, time interval and complementary examination, birth weight and necropsy, all of which presented a degree of accuracy in their filling - in far bellow that required, thus evidencing the limitations of the official death statistics based on this information.

KEWORDS: Death certificates. Medical records. Cause of death. 


\section{REFEREANCIAS BIBLIOGRÁFICAS}

1. ANTONOVSKY, A. Social class, life expectancy and overall mortality. Milbank mem. Fd. Quart., 45(2): 31-73, 1967.

2. BUTLER, $T$. et al. Causes of death in diarrhoeal diseases after rehydratation therapy: an autopsy study of 140 patients in Bangladesh. Bull. Wld Hlth Org., 65: 317-23, 1987.

3. FONSECA, L. A. M. \& LAURENTI, R. Qualidade da certidão médica de causa de morte em São Paulo, Brasil. Rev. Saúde públ., S. Paulo, 8: 21-9, 1974.

4. HAKKERT, R. Mecanismos subjacentes à relação entre mortalidade infanto-juvenil e a educação do país. Rev. bras. Est. pop., 3(2): 47-65, 1986.

5. LAURENTI, R. Causas múltiplas de morte. São Paulo, 1973. [Tese de Livre-Docência - Faculdade de Saúde Pública da USP].

6. LAURENTI, R. Alguns aspectos particulares referentes aos resultados da investigaçăo interamericana de mortalidade na infância na área do projeto de Stao Paulo, Brasil. Bol. Ofic. sanit. panamer., 89: 1-4, 1975.

7. LAURENTI, R. Doenças respiratórias como causa de morte no Municípios de São Paulo, SP (Brasil). Rev. Saúde públ., S. Paulo, 15: 353-63, 1981.

8. LAURENTI, R. \& MELLO JORGE, M. H. P. de. $O$ atestado de óbito. São Paulo, Centro da OMS para Classificação de Doenças em Portugues, 1981.

9. MANUAL da Classificaçẵo Estatística Internacional de Doenças, Lesces e Causas de Obito; revisão 1975. Sāo Paulo, Centro da OMS para Classificaçĩo de Doenças em Português/Ministério da Saúde/ Universidade de São Paulo/Organização PanAmericana da Saúde, 1978.

10. MINISTÉRIO DA SAÚDE. Secretaria Nacional de Açōes Básicas de Saúde. Divisão Nacional de Epidemiologia. Manual de instruçbes para o preenchimento da declaraçăo de obito. $2^{\star}$ ed. Brasília, Centro de Documentaçăo do Ministério da Saúde, 1976.
11. MONTEIRO, C. A. Peso ao nascer e excesso de mortalidade infantil em São Paulo. Rev. Saúde públ., S. Paulo, 14: 1-18, 1980.

12. OYA-SAWYER, D. et al. The impact of urbanization in Brazil. Wld Hith Statist. Quart., 40: 84-95, 1987.

13. PHAROAH, P. O. D. \& MORRIS, J. M. Post neonatal mortality. Epidemiol. Rev., 1: 170-83, 1979.

14. PUFFER, R. R. \& SERRANO, C. V. Garecterísticas de la mortalidad en la nitiez. Washington, D. C., Organización Panamericana de la Salud, 1973. (OPAS Publicacion Científica, 262).

15. PUFFER, R. B. \& SERRANO, C. V. Patterns of birthweights. Washington, D. C., Pan-American Health Organization, 1987. (PAHO-Scientific Publication, 504).

16. SAAD, P. M. Um método para estimar o peso dos fatores sócio-econômicos sobre a mortalidade na infância a partir de informaçoes retrospectivas das mães: aplicaç̃o para o Estado de São Paulo. In: Encontro Nacional de Estudos Populacionais, 4\%, Águas de Så Pedro, SP, 1984. Anais. São Paulo, Associação Brasileira de Estudos Populacionais, 1984. v. 2, p. 1105-39.

17. STROZZZ, G. M. et al. Estudo da causa básica de b́bito em menores de 15 anos, ocorridos em hospital de Florianópolis, SC (Brasil) em 1982. Rev. Saúde públ., S. Paulo, 19: 123-32, 1985.

18. VICTORA, C. G. et al. Epidemiologia da desigualdade. São Paulo, Hucitec, 1988.

19. VICTORA, C. G. BLANK, N. Mortalidade infantil e estrutura agrária no Rio Grande do Sul. Cienc. e Cult., 32: 1223-35, 1980.

20. ZDEB, M. Difference in trends of postneonatal mortality by birthweight in uptate New York, 1868-1979. Amer. J. publ. Hlth, 72: 734-6, 1982.

Recebido para publicação em 29/5/1989 Reapresentado em 23/111990 Aprovado para publicação em 24/1/1990 